\title{
SIMULATION OF FLUID POWER SYSTEMS IN CONSIDERATION OF UNCERTAINTIES
}

\author{
Andreas Rüdenauer \\ Karlsruhe Institute of Technology (KIT), \\ Chair of Mobile Machines (Mobima) \\ Karlsruhe, Germany
}

\author{
Dr.-Ing. Phillip Thiebes \\ Karlsruhe Institute of Technology (KIT), \\ Chair of Mobile Machines (Mobima) \\ Karlsruhe, Germany
}

\begin{abstract}
This paper presents the fundamental interrelation of uncertainty in the context of the modeling and simulation process of fluid power systems. Building up a simulation model means to represent a real system and its dynamic behavior in an experimental model. This process generally requires a model simplification. Additionally, in an early stage of product development, a certain lack of knowledge about a system is to be assumed. These so called epistemic uncertainties lead to a deviation of the simulated system's behavior from the real one. One possibility to consider such uncertainties in simulation is by using the approach of fuzzy arithmetic. This approach allows substituting crisp parameter values through fuzzy intervals. This way, uncertainty-sensitive simulation results can be obtained and the reliability of simulation can be significantly increased.

Especially for the simulation of fluid power systems with lumped parameters, a trade-off between simulation performance and the level of detail of the model has often to be accepted. The assumption of uncertainties in fluid power simulation shows the potential of creating simplified simulation models, which at the same time promise to have a high conformance to the real system behavior.

Using the fuzzy arithmetic approach under application of the transformation method by Hanss, the potential for fluid power system simulation in consideration of epistemic uncertainties is explained and evaluated. Therefore, a simulation model is tested as an example application using different degrees of parameter fuzzification.
\end{abstract}

\section{INTRODUCTION}

In the last years, simulation technology has become gradually accepted as a tool to increase the efficiency of the product development process of fluid power systems. There are many reasons which contribute to this trend. The main motivation however can be summarized by the fact that the amount of real prototypes can be reduced and therefore development time and costs are optimized [1]. Furthermore, shorter product innovation cycles and product complexity will contribute to a more intensive and integrated usage of simulation in the future [2]. One of the main drivers, which lies underneath this process of increased simulation usage, is to gain knowledge about a system and its dynamic processes, which afterwards can be transferred to reality (on the basis of [3]). To do so, models have to be created which "adequately" represent the system and which can be used for (digital) experimentation or simulation. A model is by definition the representation of the essential properties of a system. This implies the fact, that irrelevant effects for one certain observation have to be explicitly excluded from the model. Therefore, a deviation of experimentation results between a real and a simulated system always has to be assumed. This deviation can be traced back to simulation errors and uncertainties [4]. Whereas simulation errors have their origins in mathematics, or in numerics respectively, uncertainties represent the nondeterminism of a source for a result deviation. The latter can again be split up into irreducible or reducible effects, which leads to the corresponding definition of aleatory 
and epistemic uncertainties. In total, the determination of the three mentioned effects contribute to how realistic a model is.

In the area of fluid power systems, a very common way to simulate the dynamic system behavior is via physical models with lumped parameters. As a precondition for building up a model, there are two possible cases: A physical system already exists and shall be modeled or there is a, to this point of time not-exisiting system, which then has to be proposed. Taking one of these possibilities and the task, of building up a model of the system, the challenge rises that a certain level of detail has to be defined at first. And, depending on this level of detail, sooner or later one might come to the point, where the knowledge about the system is not sufficient to assign values to the model parameters. This phenomenon is often described by the term "lack of knowledge" in scientific literature (e.g. [4-6]), which again is the main reason for the definition of the above mentioned epistemic uncertainties. This type of uncertainty represents a crucial influence on the conformance of a model to an existing physical system, as they are individual for each model - whereas simulation errors are to a great part irrelevant for the modeling process. In order to explain this context in detail, a classification of uncertainties will be given as well as a discussion about the modeling process with focus on the influence of uncertainties in fluid power systems simulation.

\section{CLASSIFICATION OF UNCERTAINTIES}

Assuming uncertainties and errors in the modeling and simulation process is a key factor to assess the deviation of measurement and simulation results. This again is a basis for evaluating the potential validity of a simulation (see Fig. 1).

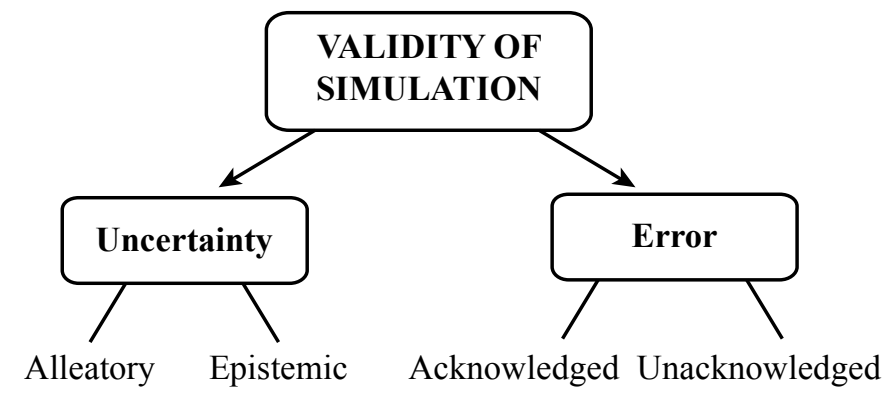

Fig. 1 - Categorization of potential simulation validity

The term simulation validity in this case is not just referred to as the validation phase of a simulation or computerized model to reality, but it furthermore is meant to cover the influence factors in all modeling phases and activities, which will contribute to a deviation of simulation and measurement results. In order to distinguish those factors, corresponding definitions will be given in this section.

In simulation, the term uncertainty is used to express that a nominal simulation is not a complete imitation of reality but lacks some unspecified parts [7]. Furthermore, two already above mentioned types of uncertainty are defined in literature in order to qualify the sources of uncertainty.
The first type is named as aleatory uncertainty (aleae [lat.] = dice), which according to Oberkampf [4] describes the inherent variation associated with the physical system or the environment under consideration. Sources of aleatory uncertainty are commonly represented by distributed quantities that can take on values in an established or known range, but for which the exact value will vary by chance. This is the reason why aleatory uncertainties are also referred to as irreducible uncertainties. Alternatively, aleatory uncertainty is denoted by the terms inherent uncertainty, variability and stochastic uncertainty. The fact, that chance is the most influencing factor on this type of uncertainty, probability density functions are normally used to propagate them in a simulation model, e.g. by Monte Carlo simulation [7, 8]. An example for aleatory uncertainties is the production tolerance, which exists in a known range but with by chance varying values.

The second type of uncertainty is described by the so called epistemic uncertainties (episteme [gr.] = knowledge). In contrast to aleatory uncertainties, they arise from a lack of information, vagueness in system definition or simplification and idealization. Oberkampf [4] defines epistemic uncertainty as a potential inaccuracy in any phase or activity of the modeling process that is due to lack of knowledge. As a consequence, the inaccuracy does not necessarily have to exist as it is a "potential inaccuracy". In literature, epistemic uncertainty is also referred to as reducible, subjective or cognitive uncertainty. The contribution of epistemic uncertainties to a system can be regarded by fuzzy numbers, interval methods or evidence theory. Although many approaches have been made up - often, approaches for FEmodels - , it has turned out, that many of them are useable for specific cases only [9]. Promising interval or fuzzy approaches come with a general applicability by being able of using blackbox models [7]. One of them is the transformation method of fuzzy arithmetic of Hanss [10], which will be regarded in this paper.

In terms of completeness of the classification, the occurring error in modeling and simulation shall be explained: an error occurs according to Oberkampf [4] as a recognizable inaccuracy which is not due to lack of knowledge and which is identifiable upon examination. It furthermore can be split up into acknowledged or unacknowledged errors. In case of the first, the analyst is aware of the error during modeling and simulation. An example is the finite precision arithmetic in a computer which at the bottom line is a rounding error. The unacknowledged errors are not recognized by the analyst, but still recognizable, e.g. if a simulation mistake occurs.

As the lack of knowledge has a striking influence on the process of building up fluid power models and thus, on the quality of simulation results, epistemic uncertainties will later be regarded more in detail in this context. In order to clarify the above mentioned influence, the following section will give a general overview about the modeling process 


\section{MODELING PROCESS}

There are many possibilities to define the activities and phases of the modeling process. On the one hand, for a very specialized modeling task, it is valid to use a general procedure for problem solving (e.g. S.P.A.L.T.E.N. [11]). On the other hand, many procedure models have been made up especially for the modeling and simulation process, which cover a broad range of application cases and modeling objectives. Depending on the latter, the number of necessary activities and modeling phases increases with the specificity of the application case. A fundamental model for the modeling and simulation process was published by the Technical Committee on Model Credibility of the Society for Computer Simulation [12] in 1979 (see Fig. 2). It generally introduces three modeling levels "reality", "conceptual model" and "computerized model", which interdepend by the modeling phases for qualification, verification and validation of the models and the corresponding consecutive activities, "Analysis", "Programming" and "Computer Simulation".

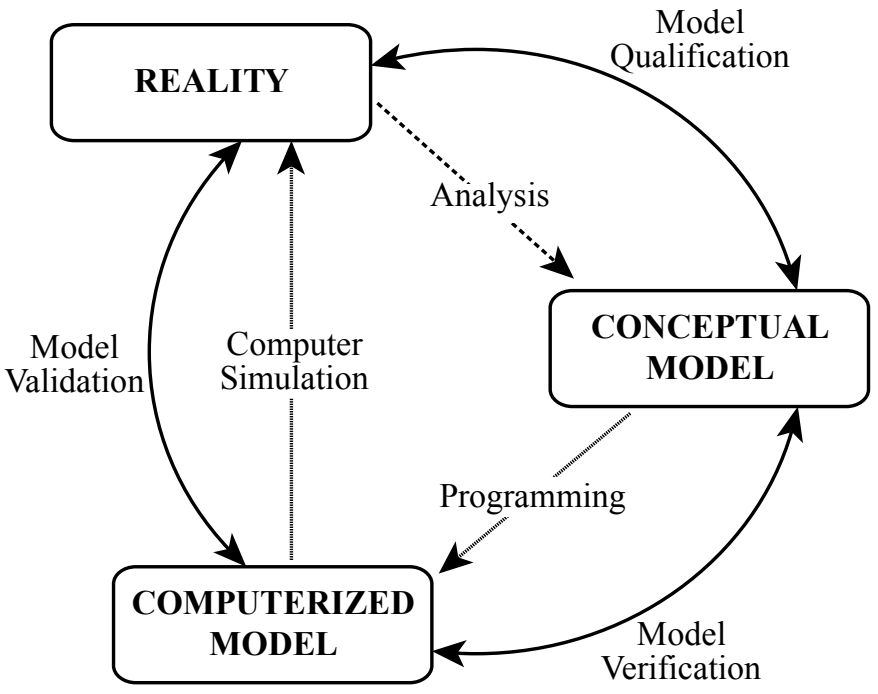

Fig. 2 - Modeling procedure proposed by the Society for Computer Simulation [12]

More detailed and elaborated models were published e.g. by Jacoby and Kowalik [13], who had their focus on mathematical modeling aspects, as well as the modeling effort. Sargent, for example, presented a modeling procedure, which refines the model in Fig. 2 with the objective of a general applicability for any modeling and simulation process [14].

The so far presented modeling procedures have their specific focus concerning a specific modeling and simulation objective. For regarding the error and uncertainty in modeling and simulation, Oberkampf et al. in [4] propose a six phasemodeling procedure for computational modeling and simulation (see Fig. 3) based on existing, partly in this paper mentioned modeling procedures. In his modeling procedure, he focuses, amongst others, on the distinction between aleatory and epistemic uncertainties. Therefore, the proposed procedure of Oberkampf will be explained more in detail.

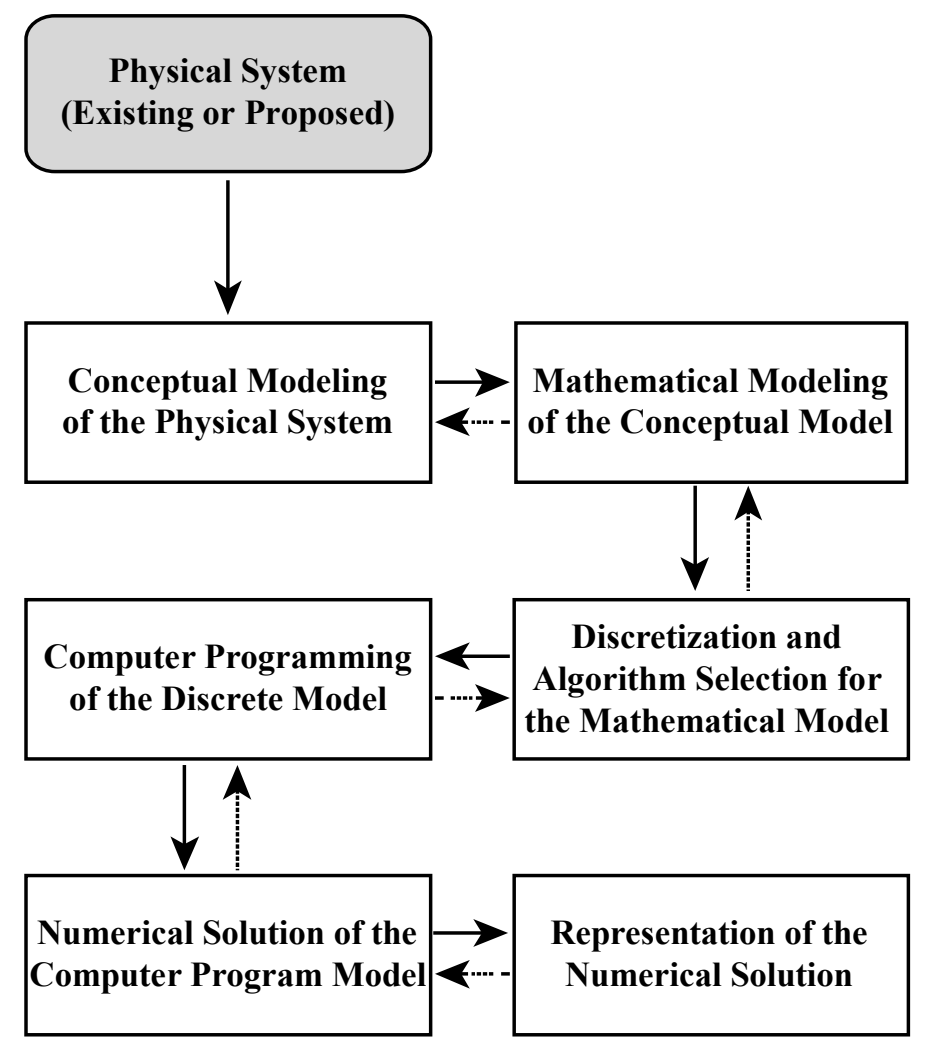

Fig. 3 - Modeling phases and activities by Oberkampf [4]

The first step of the procedure is the derivation of a conceptual model based on an existing or a proposed physical system or process. In this phase, the simulation objectives, the specification of the physical system and the environment as well as boundary conditions are set. This includes physical events and the concept for coupling of different physical processes, which in fluid power systems often is the coupling of hydraulic and mechanical structures. Also, the modeling and simulation effort is defined and in dependency on that, a required level of detail for the model is determined. The complexity of the model afterwards will depend on the number of physical phenomena being considered and the complexity of those phenomena. On basis of the conceptual design of the model, the system and environment characteristics have to be analyzed concerning the existence of aleatory or epistemic uncertainty. The first phase, however, does explicitly exclude the creation of mathematical equations. The propagation or the mathematical representation of uncertainties also will take place in the next phase.

The second phase, mathematical modeling of the conceptual system, contains the development of detailed mathematical models of the conceptual models as a main activity. This includes the complete specification of all differential equations, auxiliary, boundary and initial conditions of the system. In the total modeling and simulation procedure, 
the amount of nondeterministic elements, which lead to uncertainties is especially high in the phase of mathematical modeling. Aleatory uncertainties might typically exist through the definition of system parameters, boundary or initial conditions, if the values vary randomly from element to element. Epistemic uncertainties exist just by the fact that a mathematical model is always incomplete as it is a simplification of reality.

The following phases,

- discretization and algorithm selection for the mathematical model,

- computer programming of the discrete model,

- numerical solution of the computer program model and

- $\quad$ representation of the numerical solution

are neither influenced by values which vary by chance nor by lack of knowledge. As a consequence, the quality of the activities in these phases is mainly controlled by simulation errors, which to a great part have a high level of maturity and therefore are of minor importance for the following examination.

\section{UNCERTAINTY IN THE MODELLING PROCESS OF FLUID POWER SYSTEMS}

There are in general two approaches for the simulation based analysis of a hydraulic system in the time domain [15]:

- exact calculation of (mass or volume) flow through components using computational fluid dynamics (CFD),

- $\quad$ simulation of hydrostatic systems on the basis of models with lumped parameters.

The latter nowadays dominates the application area of complete system simulations resulting from the ease of model build up. In this paper, the focus of simulation of fluid power systems is on simulation with lumped parameters. In this type of simulation, usually predefined components, which represent a physical phenomenon, can be chosen from a model library and afterwards are interconnected via nodes [16]. Additionally, at least one hydraulic capacity has to exist between two components, which in dependency of the simulation tool may be already included as a property of the node or which also may be a separate model component. The basic condition in a hydraulic capacity is the balance equation of the hydraulic flow $Q$.

$$
\sum_{i} Q_{i}=0
$$

where $i$ corresponds to the incoming or outgoing volume flows. For correlation with the system pressure, the pressure build up equation for a hydraulic capacity is used, which is:

$$
\dot{p}=\frac{1}{C_{H}} \sum_{i} Q_{i}
$$

where $\dot{p}$ represents the pressure change over time and $C_{H}$ represents the constant of the hydraulic capacity. This way, a hydraulic network can be modeled.

One of the main challenges in fluid power systems modeling with lumped parameters in contrast to other domains can be reasoned by the functional structure of a hydraulic model (see Fig. 4). This can be explained using the Contact and Channel (C\&C) Meta-Modelling approach, which allows the consideration of system functions in reference to a component's form [17]. A system function can be fulfilled according to the C\&C model, if at least two working surface pairs (WSP), which are the pair-wise interfaces between components and its environment, are interconnected via a channel and support structure (CSS), which transfers or stores the system variables energy, material or information. In hydraulics, two hydraulic components, e.g. two hydraulic cylinders connected via a hydraulic line, fulfill a fluid power system function through the

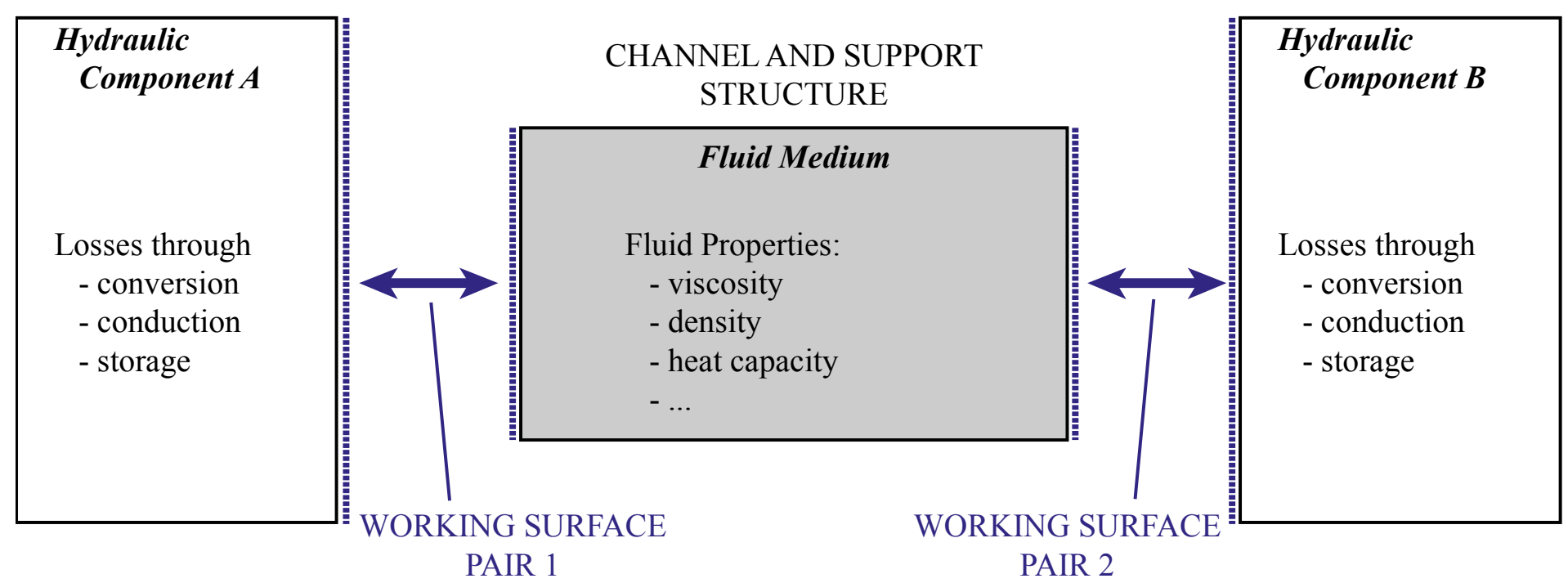

Fig. 4 - Basic Functional Structure of a Hydraulic Model 
interconnection of the corresponding WSPs, piston and oil, with a channel and support structure, the fluid, in this case oil. In dependence on the application case, the fluid is able to carry over many system functions in a fluid power system, which can be divided up into main functions, e.g. energy or signal transfer, and auxiliary functions, e.g. lubrication, washing out of particles or heat transfer. From a system engineering point of view, the fluid enables the fluid power system, thus, to cover energy-, material- and information-flows. Additionally, these functions can be easily realized in hydraulics, which contributes to the fact, that they are commonly applied in fluid power systems and therefore, the modeling of the fluid medium and its interactions comes in for certain modeling challenges - in contrast e.g. to electrical current regarded as a contact and channel structure.

The fluid, in this case oil, has many properties - e.g. viscosity, heat capacity or density -, which are influenced by the temporary system function, which the fluid takes over. Additionally, depending on the environmental and operating condition of the system, the state of these properties can vary quickly over time. This leads to increased requirements for the modeling process. In the first modeling phase of Oberkampf's modeling procedure - the conceptual modeling of a physical model -, a clear definition of which effects and which system state(s) are to be considered for simulation is striking for the quality and validity of simulation results. Based on this scenario abstraction, the decision can be derived on which modeling components have to be used in which level of detail. This directly is in correlation with the quantity of epistemic uncertainty. The defined system and environment specification, for example, requires a distinct amount of knowledge which might or might not exist.

In the second modeling phase of Oberkampf, the mathematical models are created. The concept of modeling a fluid power system with lumped parameters is furthermore influenced by the experience, that certain properties are dominant. In case of flown through components as tubes, hoses, valves, throttles, the greatest influence is induced by the flowing fluid. This leads predominantly to a mathematical description of the models by the pressure losses, which result from flow- and friction- properties. In terms of the predictability of a fluid power model's "realistic" behaviour, the parameterization of each model has to be regarded in context of epistemic uncertainty depending on the "amount" of lack-ofknowledge. Many simulation tools offer various possibilities to model a single aspect of a problem. This is provided for the fact that a fluid power phenomenon can be modeled with alternative models (or submodels) and that a wide range of predefined models already exists. A complete physical system can thus be modeled on various ways. The objective to reach high prediction capacity of a fluid power simulation, however, does not necessarily come with a highly detailed model under consideration of all existing phenomena. It rather can be achieved by identifying the dominant controlling factors of a system and their influences correctly. Within this context, the consideration of epistemic uncertainties will help a lot to increase the ability to make proper predictions. In this case, this shall be achieved by the usage of fuzzy-valued parameters which assume the worst-case deviation of those dominant controlling factors as uncertainty.

In order to analyze the propagation of epistemic uncertainties in a fluid power simulation system with fuzzyvalued parameters, the transformation method (TM) of Hanss will be used. An overview of the necessary steps and the working procedure of the TM will be presented in the following section.

\section{THE TRANSFORMATION METHOD OF HANSS}

The transformation method (TM) of fuzzy arithmetic of Hanss is a practical implementation of Zadeh's extension principle, which was published in 1975 [18-20] and which basically states that any fuzzy arithmetical computation can be solved by a multi-dimensional optimization problem.

The TM uses fuzzy-parameterized numbers to model epistemic uncertainties and calculates a fuzzy-valued output of a dynamic plant model. Very common for the modeling of a fuzzy number $\tilde{p}$, is the application of triangular fuzzy numbers. They are denoted as

$$
\tilde{p}=\operatorname{tfn}\left(\bar{x}, w_{l}, w_{r}\right)
$$

where $\bar{x}$ is the nominal value, $w_{l}$ and $w_{r}$ the left-hand and right-hand worst-case deviation. The fuzzy number can then be expressed by a membership function $\mu_{\tilde{p}}$, which can be interpreted as quantification of the possibility that a specific interval occurs. Fig. 5 shows an exemplary graph of a triangular fuzzy number.

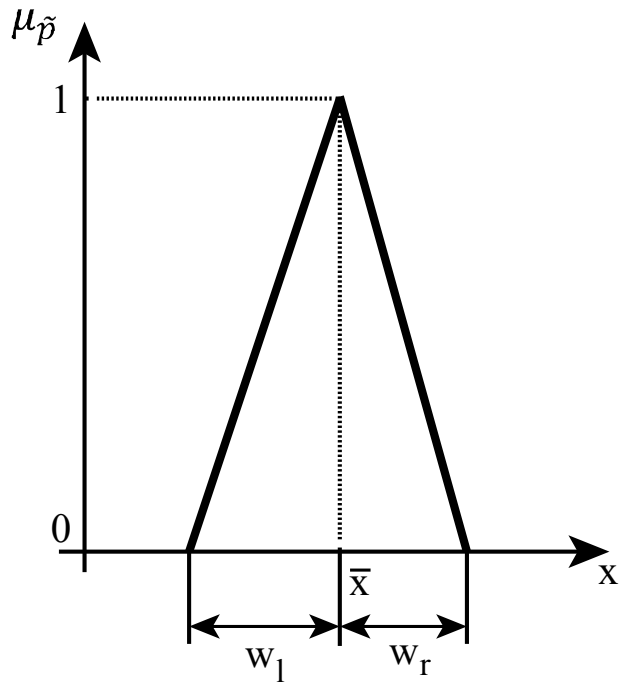

Fig. 5 - Triangular fuzzy number

The fuzzy arithmetic approach can be applied on a simulation model represented as a black-box and has been published so far for many finite elements-based problems (e.g. 
[21]), particle problems ( e.g. [22]) as well as its application to multi body systems (e.g. [5]).

The TM takes a five step procedure, which is here presented in short on the basis of [23]. These five steps are for a system with $n$ presumed uncertain, fuzzy-parameterized input parameters $\tilde{p}_{i}, i=1,2, \ldots, n$ and $\mathrm{N}$ fuzzy-parameterized output parameters $\tilde{q}=f\left(\tilde{p}_{1}, \tilde{p}_{2}, \ldots, \tilde{p}_{N}\right)$.

1. Decomposition of the input fuzzy numbers according to $m$ equally-spaced intervals of width $\Delta \mu$ and the corresponding $(m+1)$ levels of membership $\mu_{j}=\frac{j}{m}$, leading to a set $P_{i}$ of intervals $X_{i}^{j}$ of the form

$$
\begin{gathered}
P_{i}=\left\{X_{i}^{(0)}, X_{i}^{(1)}, \ldots, X_{i}^{(m-1)}, X_{i}^{(m)}\right\} \\
\text { with } X_{i}^{(j)}=\left[\begin{array}{c}
\left.a_{i}^{(j)}, b_{i}^{(j)}\right], a_{i}^{(j)} \leq b_{i}^{(j)} \text { and } \\
j=0,1, \ldots, m
\end{array}\right.
\end{gathered}
$$

2. Transformation of the input intervals $X_{i}^{j}$ to arrays $\hat{X}_{i}^{j}$ :

$$
\hat{X}_{i}^{(j)}=\left({ }^{1} \hat{x}_{i}^{(j)},{ }^{2} \hat{x}_{i}^{(j)}, \quad \ldots,{ }^{r} \hat{x}_{i}^{(j)}\right)
$$

with

$$
\begin{array}{ll}
r=2^{n} & \text { for reduced TM } \\
r=(m+1-j)^{n} & \text { for general TM }
\end{array}
$$

Depending on the monotonicity of the simulation problem, the TM can be applied in a general, a reduced, and an extended form, see Hanss $([10,24,25])$.

3. Evaluation of the system model in the decomposed and transformed form by the output arrays $\hat{Z}^{(j)}$

$\hat{Z}^{(j)}=\left({ }^{1} \hat{Z}^{(j)},{ }^{2} \hat{Z}^{(j)}, \ldots,{ }^{k} \hat{Z}^{(j)}, \ldots,{ }^{r} \hat{Z}^{(j)}\right)$,

where the $k$ th element of the Array is given with

$$
{ }^{k} \hat{z}^{(j)}=f\left({ }^{k} \hat{x}_{1}^{(j)},{ }^{k} \hat{x}_{2}^{(j)}, \ldots,{ }^{k} \hat{x}_{n}^{(j)}\right)
$$

4. Retransformation of the output arrays $\hat{Z}^{(j)}$ in intervals $Z^{(j)}$ :

$Q=\left\{Z^{(0)}, Z^{(1)}, \ldots, Z^{(m-1)}, Z^{(m)}\right\}$

with $\quad Z^{(j)}=\left[c^{(j)}, d^{(j)}\right] \quad$ for each

membership level $\mu_{j}$.

5. Recomposition of the output intervals to fuzzy-valued output parameters $\tilde{q}$.

\section{EXAMPLE APPLICATION}

In order to demonstrate the effect of assuming epistemic uncertainty via the TM, in the following an example of typical modeling challenges in the field of fluid power systems modeling and simulation will be given. As application, a hydraulically preloaded hydropneumatic suspension system is chosen.

According to the modeling phases of Oberkampf, as presented in figure 3 , the first step for modeling in consideration of uncertainties is the built up of a conceptual model. This can be achieved, e.g. by a schematic diagram, as it can be seen for the hydropneumatic suspension system in figure 6. For the preparation of a simulation model, it is essential to set the simulation objectives and the quantity and quality of phenomena which are to be considered. In order to clarify the effect of uncertain modeling and simulation, the focus of observation will be restricted to the uncertain modeling of the hydraulic lines in this case.

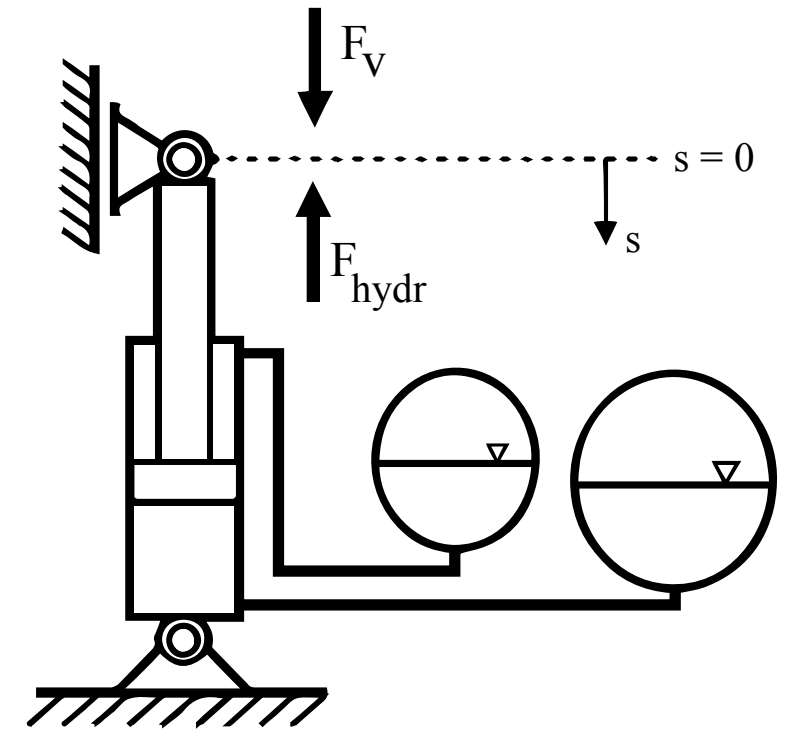

Fig. 6 - Schema of a hydropneumatic suspension

In terms of functional assignment of the hydraulic lines, the system boundaries are set by the hydraulic-hydraulic WSP at the cylinder chamber and the hydraulic-hydraulic WSP at the hydraulic accumulator. These WSPs then are physically interconnected by the CSS, the hydraulic line itself. The model of the hydraulic line shall consider the functional behavior of hydraulic capacity on the one hand, which is capable of storing oil, and on the other hand of hydraulic resistance, which leads to a pressure loss depending on the flow rate. Thermal behavior will be neglected.

Depending on the modeling specification, the existence of uncertainties has to be analyzed. In order to keep the application example simple, the uncertain influences will be reduced in this paper to selected influences of the oil and the pressure losses. The latter will depend on the flow rate. A significant influence parameter has to be identified in the next step, when the mathematical modeling will be regarded. The 
uncertainty induced by the model of the oil is influenced by many factors again. The fact that the status and the exact type of oil might be unknown in an early point of the potential development process as well as the exclusion of thermal behavior contributes to an increasing model uncertainty. For the oil, therefore the bulk modulus $E_{\text {Oil }}$ and the density $\rho$ are chosen as representative uncertain parameters. It is again to be underlined that the selection of the uncertain parameters of a model always has to be done individually for each model and each simulation objective.

In the next step, the mathematical model will be derived from the conceptual one. In the most basic variant, the mathematical model consists of the pressure build up equation as connection between hydraulic capacity nodes (see eq. 2) and of hydraulic resistences, which are represented by orifices. In the model, it has to be considered that hydraulic lines consist of tubes and hoses. The parameterization of the system has to be carried out with substitute values.

The behavior of the orifices is described by the orifice formula.

$$
Q=\alpha_{D} \cdot A \cdot \sqrt{\frac{2 \cdot \Delta p}{\rho}}
$$

Where $\alpha_{D}$ represents the flow coefficient and $A$ represents the cross-sectional area of the orifice. Corresponding to the assumption above, two types of orifices have to be chosen: orifices which are to represent tubes and orifices which are to represent hoses. The criterion to express the different flowcharacters is set to the cross-sectional area of the orifice or by a so called substitute diameter $d$, respectively. As hoses have a higher friction, the substitute diameter of the cross-sectional area is set smaller than the one of the tubes. The flow coefficient is held constant for both types. The volume of the hydraulic capacities is set according to the designated model representation, which again corresponds to the respective volume section for hoses or tubes. The ranges of the worst-case deviations for uncertainty in both cases are estimated as a deviation of approx. $15 \%$ from the nominal value. This is a rough assumption, as there are no measurement results given so far. These deviations due to uncertainty are expressed via triangular fuzzy numbers. The length of the used tubes is in total $1.5 \mathrm{~m}$. The length of hoses is $0.5 \mathrm{~m}$.

Additionally, the influence of epistemic uncertainty created by the fluid, which is a HLP-oil, shall be taken into account. The main drivers for considering the oil-induced uncertainty in relation with the system dynamics are here regarded as the density and the bulk modulus. The appearing uncertainty concerning the oil density is mostly influenced by temperature and the air, which is solved in the oil. For the bulk modulus, it is important to know, that in this case, the simulation model allows the definition of one constant bulk modulus. As there are many dependencies, as pressure, air in oil, the inclusion of an uncertain factor via a fuzzy parameter shall cover this uncertainty and this way, create a "real" equivalent bulk modulus. For the determination of the worst-case deviations, it is also relevant to notice, that e.g. increasing temperature and dissolved air in the liquid decreases the bulk modulus, whereas increasing pressure increases the bulk modulus.

Consecutively, there is a wide deviation to consider. The values of the worst-case deviation-ranges were chosen for each parameter due to the acting or assumed loads and pressures in the system based on elementary hydraulic literature [27, 28]. The resulting fuzzy numbers are also implemented as triangular fuzzy numbers.

Table 1 gives an overview of an exemplary parameterization of the fuzzy values for the triangular fuzzy numbers with an assumed nominal value and the corresponding left- and right-hand worst-case deviation. These values later will be used for simulation.

\section{Table 1 - Fuzzy representation of uncertain system parameters}

\begin{tabular}{l|r|r|r}
\hline Parameter $\widetilde{\boldsymbol{p}}=\boldsymbol{t} \boldsymbol{f n}\left(\overline{\boldsymbol{x}}, \boldsymbol{w}_{\boldsymbol{l}}, \boldsymbol{w}_{\boldsymbol{r}}\right)$ & \multicolumn{1}{c|}{$\boldsymbol{x}$} & \multicolumn{1}{c|}{$\boldsymbol{w}_{\boldsymbol{l}}$} & \multicolumn{1}{c}{$\boldsymbol{w}_{\boldsymbol{r}}$} \\
\hline $\begin{array}{l}\text { substitute diameter for tubes } \tilde{d}_{t} \\
{[\mathrm{~mm}]}\end{array}$ & 8 & 1.5 & 1.5 \\
\hline $\begin{array}{l}\text { substitute diameter for hoses } \tilde{d}_{h} \\
{[\mathrm{~mm}]}\end{array}$ & 6.5 & 2 & 2 \\
\hline $\begin{array}{l}\text { density } \tilde{\rho} \\
{\left[\mathrm{kg} / \mathrm{m}^{3}\right]}\end{array}$ & 850 & 50 & 10 \\
\hline $\begin{array}{l}\text { bulk modulus } \tilde{E}_{\text {Oil }} \\
{[\mathrm{bar}]}\end{array}$ & 17.000 & 5.000 & 1.000 \\
\hline
\end{tabular}

The complete model as described above is built up in AMESim ${ }^{\circledR}$ and then simulated in a co-simulation with Matlab ${ }^{\circledR}$ using the GUSMA standard interface for cosimulation [26]. The five steps of the TM were integrated in the toolbox FAMOUS (Fuzzy Arithmetical Modeling Of Uncertain Systems) by the Institute of Engineering and Computational Mechanics at the University of Stuttgart. FAMOUS is based on Matlab ${ }^{\circledR}$ and will be used for the evaluation of the example [7].

The system in this example was actuated with a sinusoidal track profile from the ground $(s=0)$ in Fig. 6 . The resulting displacement path $s$ with crisp parameterization consecutively also has sinus form. The result after the above mentioned fuzzification of parameters is shown qualitatively in Fig. 7. The variation of the color is an indicator for the membership level of the corresponding fuzzified output parameter. The darker the color, the higher is the possibility that the output parameters occurs at that point. For each time step, a membership level of the output parameter is given.

Due to the decomposition of fuzzy input numbers in the TM, an additional result part of a fuzzy arithmetic calculation is the sensitivity analysis of all fuzzified input parameters for each output parameter. It can be expressed by its absolute or relative sensitivity. This can be seen for the force output in Fig. 8. It can be noticed that the influence of the uncertain density parameter is comparatively high - both in relative and in absolute perspective. 


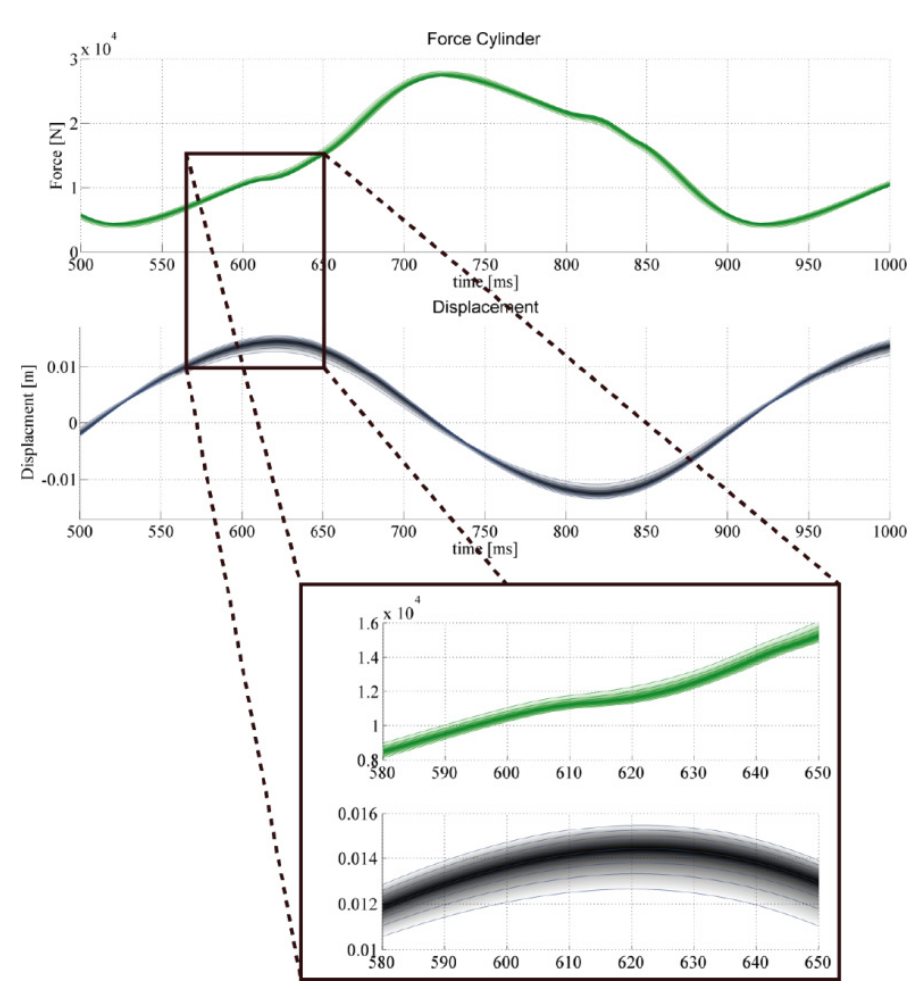

Fig. 7 - Fuzzy-valued force of cylinder and displacement of piston
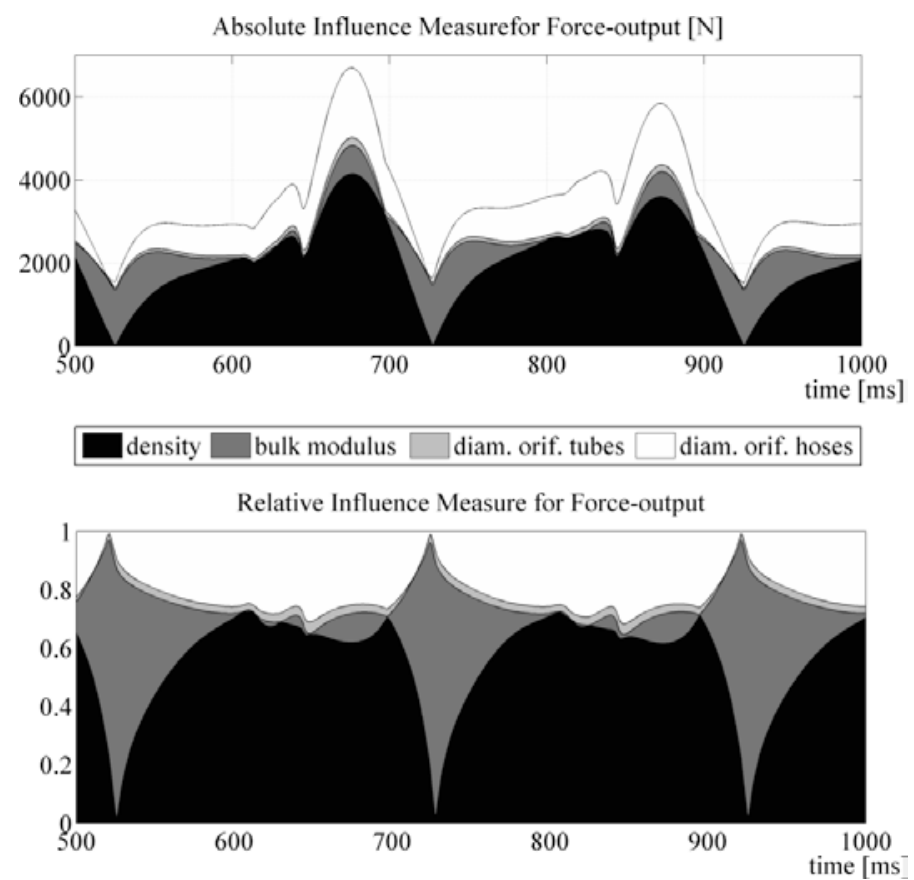

Fig. 8 - Absolute and relative uncertainty measures for Force of cylinder

\section{DISCUSSION}

The results in Fig. 7 show an extraction of the fuzzified output of the simulation. Also Fig. 8 shows the relative influence of each fuzzy parameter on the output.

In comparison with the crisp-valued result it can be seen, that in this parameter case, the fuzzy-output does not have a wide deviation. As a consequence, the range of epistemic uncertainty, which was given through the fuzzified input parameters, does not have a striking influence on the system deviation. Of course, the ability of predicting the real systems's behavior with a fuzzified simulation result is directly affected by the worst-case assumption and how realistic the latter is. Exaggerated worst-case deviation at the input would lead to extreme output ranges. Using substitute models, as it is common in fluid power simulation, it is until today inevitable to apply the experience of an analyst or engineer for the modeling and simulation process. The implication of epistemic uncertainty however is a useful method to improve the validity and quality of simulation results and cover the real system's potential.

\section{CONCLUSION AND OUTLOOK}

Simulation of fluid power systems with lumped parameters has been established as a tool to increase efficiency in product development. One main challenge in the modeling and simulation process is represented by the lack of knowledge about the system and its boundary conditions due to high system complexity or simplifications, which increase the effort of correct parameterization. The latter given as an important reason for the deviation of the simulated system's behavior to the real one, it is important for the quality and validity of simulation results to be aware of simulation errors and uncertainties.

The consideration of epistemic uncertainty is promising, if lack of knowledge is the main reason for the deviation of simulation from reality. Especially in the conceptual and mathematical modeling phase, epistemic uncertainties are vitally important und were evaluated in this paper in terms of their appearance in modeling of fluid power systems.

Using fuzzy arithmetics, one possibility to analyze the propagation of epistemic uncertainties is via the transformation method by Hanss. It was shown, that depending on the implemented worst-case deviation of input parameters the output variables vary.

Until now, the parameterization of fuzzy values is a empirical process due to long simulation times and many recursions. This process of parameterization still shows potential for optimization.

The parameter sensitivity analysis, which is a result of the transformation method, shall be evaluated in the future to derive consequences and recommendations for action for model simplification. 


\section{NOMENCLATURE}

CFD
C\&C
WSP
CSS
TM
$Q$
$\dot{p}$
$C_{H}$
tfn
$\bar{x}$
$w_{l}$
$w_{r}$
$\mu_{\tilde{p}}$
$\left.\tilde{C}^{\cdot}\right)$
$\tilde{p}_{i}$
$\tilde{q}_{r}$
$m$
$P_{i}$
$X_{i}^{j}$
$\hat{X}_{i}^{j}$
${ }^{k} \hat{x}_{i}^{(j)}$
$Q_{r}$
$\hat{Z}^{(j)}$
$k_{\hat{Z}}$
$\rho$
$E_{O i l}$
$A$
$\alpha_{D}$

Computational Fluid Dynamics

Contact and Channel

Working Surface Pair

Channel and Support Structure

Transfer Method

Hydraulic volume flow

pressure change over time

constant for hydraulic capacity

triangular fuzzy number

nominal value of the fuzzy parameter $\tilde{p}$

left-hand worst-case deviation

right-hand worst-case deviation

membership level of the fuzzy number $\tilde{p}$

fuzzy-valued quantity

fuzzified-valued input

fuzzified-valued output

decomposition number

set of decomposed intervals representing $\tilde{p}_{i}$

one element of $P_{i}$

parameter array of the TM associated to $P_{i}$

one element of $\hat{X}_{i}^{j}$

set of decomposed intervals representing $\tilde{q}_{r}$

parameter array of the TM associated to $Q_{r}$

one element of $\hat{Z}^{(j)}$

density

bulk modulus

cross-sectional area of orifice

flow coefficient of orifice

\section{ACKNOWLEDGMENTS}

The authors would like to thank the Institute of Engineering and Computational Mechanics at the University of Stuttgart for the support and the provision of the fuzzyarithmetic toolbox FAMOUS.

\section{REFERENCES}

[1] Simulation in der Fluidtechnik. $O+P .562-571,2006$

[2] Abele, E., and Reinhart, G., 2011, "Zukunft der Produktion : Herausforderungen, Forschungsfelder, Chancen", München, Germany.

[3] VDI-Guideline 3363, 2000: "Simulation von Logistik-, Materialfluß- und Produktionssystemen Grundlagen".

[4] Oberkampf, W. L., DeLand, S. M, Rutherford, B. M., Diegert, K. V. and Alvin, K. F., 2002 "Error and uncertainty in modeling and simulation," Reliability Engineering and System Safety, vol. 75, pp. 333 - 357.

[5] Walz, N.-P., Firscher, M., Hanss, M. and Eberhard, P., 2012, "Uncertainties in multibody systems - potentials and challenges.," in 4th International Conference on
[6]

Uncertainty in Structural Dynamics (USD), Leuven, Belgium.

Albers, A., Ebel, B. and Lohmeyer, Q., 2012, "Systems of Objectives in Complex Product Development," in Ninth International Symposium on Tools and Methods of Competitive Engineering, TMCE, p. 12, Karlsruhe, Germany.

Haag, T., 2012, "Forward and Inverse Fuzzy Arithmetic for Uncertainty Analysis with Applications to Structural Mechanics," Institut für Angewandte und Experimentelle Mechanik, Universität Stuttgart, Stuttgart, Germany.

Coyette, J.-P., 2005, "Sources of uncertainties in vibroacoustic simulations," in Symposium on Managing uncertainties in noise measurements and predictions, Le Mans, pp. 26-32.

Moens, D. and Hanss, M., 2011, "Non-probabilistic finite element analysis for parametric uncertainty treatment in applied mechanics: Recent advances.," Finite Elements in Analysis and Design, vol. 47, pp. 416.

Hanss, M., 2005, Applied Fuzzy Arithmetic - An Introduction with Engineering Applications. Berlin, Germany.

Albers, A. and Braun, A., 2011, "A generalized framework to compass and to support complex product engineering processes," International Journal of Product Development, vol. 15, pp. 6-25.

Schlesinger, S., 1979, "Terminology for model credibility," Simulation Modelling Practice and Theory, vol. 32.

Jacoby, S. L. S., and Kowalik, J. S., 1980, Mathematical Modeling with Computers: PrenticeHall.

[14] Sargent, R. G., 2007, "Verification and Validation of Simluation Models," in Winter Simulation Conference.

[15] Dronka, S., 2004, "Die Simulation gekoppelter Mehrkörper- und Hydraulik-Modelle mit Erweiterung für Echtzeitsimulation," Dissertation, TU Dresden, Dresden, Germany.

[16] Beater, P., 1999, Entwurf hydraulischer Maschinen: Modellbildung, Stabilitätsanalyse und Simulation hydrostatischer Antriebe und Steuerungen: SpringerVerlag $\mathrm{GmbH}$.

[17] Albers, A., Braun, A., Clarkson, P J., Enkler, H.-G., Wynn, D., 2009, "Contact and Channel Modelling to Support Early Desig nof Technical Systems," in International Conference of Engineering Design ICED, Stanford, CA, USA.

[18] Zadeh, L. A., 1975, "The Concept of a Linguistic Variable and its Application to Approximate Reasoning - I," Information Sciences, vol. 8, pp. 199249.

[19] Zadeh, L. A., 1975, "The Concept of a Linguistic Variable and its Application to Approximate 
Reasoning - II," Information Sciences, vol. 8, pp. 301357.

[20] Zadeh, L. A., 1975, "The Concept of a Linguistic Variable and its Application to Approximate Reasoning - III," Information Sciences, vol. 9, pp. 4380.

[21] Pratellesi, S. T. A., Haag, T., Scippa, A. and Baldanzini, N., 2011, "On the effect of terting uncertainties in the homologation tests of motorcycle helmets according to ECE 22.05," International Journal of Crashworthiness, vol. 16, pp. 523-536.

[22] Fleissner, F., Haag, T., Hanss, M. and Eberhard, P., 2009, "Uncertainty analysis for a particle model of granular chute flow," Computer Modeling in Engineering \& Sciences, vol. 52, pp. 181-196.

[23] Hanss, M. and Haag, T., 2011, "Eine Methode zur Fluid-Struktur-Simulation unter Einbeziehung von Unsicherheiten," in SIMPEP - Kongress für Simulation im Produktentstehungsprozess, Proceedings, pp. 435455, Veitshöchsheim, Germany.
[24] Hanss, M., 2002, "The transformation method for the simulation and analysis of systems with uncertain parameters," Fuzzy Sets and Systems, vol. 130, pp. 277-289.

[25] Hanss, M., 2003, "The extended transformation method for the simulation and analysis of fuzzyparameterized models," International Journal of Uncertainty, Fuzziness and Knowledge-Based Systems, vol. 11, pp. 711-723.

[26] Rüdenauer, A. and Geimer, M., 2012, "CrossCompany System Simulation using the GUSMAStandard for Co-Simulation," SIMPACK News, pp. 2630.

[27] Findeisen, D., 2006, Ölhydraulik : Handbuch für die hydrostatische Leistungsübertragung in der Fluidtechnik, 5.ed.

[28] Mang T. and Dresel, W., 2007, Lubricants and Lubrication: Wiley. 